\title{
Variable Exponent Sobolev Spaces and Regularity of Domains
}

\author{
Przemysław Górka ${ }^{1}$ - Nijjwal Karak ${ }^{2}$ - Daniel J. Pons ${ }^{3}$ \\ Received: 10 February 2019 / Published online: 20 August 2020 \\ (c) The Author(s) 2020
}

\begin{abstract}
We study the embeddings of variable exponent Sobolev and Hölder function spaces over Euclidean domains, providing necessary and/or sufficient conditions on the regularity of the exponent and/or the domain in various contexts. Concerning the exponent, the relevant condition is log-Hölder continuity; concerning the domain, the relevant condition is the measure density condition.
\end{abstract}

Keywords Sobolev spaces $\cdot$ Sobolev embedding $\cdot$ Measure density condition

Mathematics Subject Classification 46E35 - 46E30

\section{Introduction}

In Euclidean domains, variable exponent Lebesgue-Sobolev and Hölder spaces have been intensively studied during the last years (see the books $[3,5]$ for a gentle introduction, and [6] for an overview on the history of the subject). These spaces of functions provide a useful tool for the description of non-linear phenomena in elastic mechanics [21], fluid mechanics [18] and image restoration [16], for example. In some descriptions of the aforementioned phenomena, functionals of Dirichlet type with variable

$凶 \quad$ Przemysław Górka

p.gorka@mini.pw.edu.pl

Nijjwal Karak

nijjwal@gmail.com

Daniel J. Pons

dpons@unab.cl; pons.dan@gmail.com

1 Department of Mathematics and Information Sciences, Warsaw University of Technology, Ul. Koszykowa 75, 00-662 Warsaw, Poland

2 Discipline of Mathematics, Indian Institute of Technology Indore, Indore 453552, India

3 Departamento de Matemáticas, Facultad de Ciencias Exactas, Universidad Andres Bello, República 498, Santiago, Chile 
exponent are used; since the regularity of the critical points of those functionals is relevant, the embedding or inclusion problem between these type of spaces must be studied in depth. Note that this theory has also been developed in Riemannian manifolds $[9,10]$, and also in metric-measure spaces $[8,12,17]$.

In this work, we consider inclusions between Lebesgue, Sobolev, and Hölder spaces with variable exponent on Euclidean domains, and obtain sufficient and/or necessary conditions on the regularity of the exponent and/or on the domain.

In Sect. 2, we provide a brief description of variable exponent Sobolev and Hölder spaces in Euclidean domains, together with some of their elementary properties. To have a point of reference, we overview the problem concerning the density of smooth functions in variable and classical (or constant exponent) Sobolev spaces; in the variable exponent case, at least when the domain is regular enough, the regularity of the exponent is fundamental: the log-Hölder regularity turns out to be a sufficient condition.

Then we move to the subject of this work, the inclusion or embedding problem between spaces of functions. When the exponents are constant, there are many results concerning necessary and/or sufficient conditions on the regularity of the domain to achieve the embeddings [1,11]; in the variable exponent case, and at least when the domain is regular enough, the log-Hölder regularity of the exponent appears as a sufficient condition again. To clarify our contribution, we describe the (nowadays) canonical notions of regularity for domains and exponents in detail, and quote the known results for embeddings between Sobolev and/or Hölder spaces using these notions. After this has been done, we state our main results, that could be seen as a mixture of sufficient conditions on the regularity of the exponent, and necessary conditions on the regularity of the domain. The proofs of these results are given in Sect. 3.1.

In Sect. 4, we infer some consequences of our main results, providing generalizations and different proofs of some results to be found in [1].

\section{Preliminaries}

In this work, $\mathcal{L}^{n}$ is the $n$-dimensional Lebesgue measure, and $\Omega$ is a Lebesgue measurable subset of $\mathbb{R}^{n}$. If $A$ is a $\mathcal{L}^{n}$-measurable subset of $\Omega$, we write $|A|$ instead of $\int_{A} d \mathcal{L}^{n}(x)=\mathcal{L}^{n}(A)$. A variable exponent, or simply an exponent, is a bounded $\mathcal{L}^{n}$-measurable function $p: \Omega \rightarrow[1, \infty[$, usually written as $p(\cdot)$. For such a $p(\cdot)$, whenever $A$ is a $\mathcal{L}^{n}$-measurable subset of $\Omega$ define

$$
p_{A}^{+}:=\operatorname{ess} \sup \{p(x): x \text { in } A\} \text { and } p_{A}^{-}:=\operatorname{essinf}\{p(x): x \text { in } A\} .
$$

If $A=\Omega$, we simply write $p^{+}$and $p^{-}$, respectively.

In this context, the generalized Lebesgue space $L^{p(\cdot)}(\Omega)$ is the vector space of measurable functions $u: \Omega \rightarrow \mathbb{R}$ for which the functional

$$
\rho_{p(\cdot)}(u):=\int_{\Omega}|u(x)|^{p(x)} \mathrm{d} \mathcal{L}^{n}(x),
$$


is finite. The functional $\rho_{p(\cdot)}$ is convex, and $L^{p(\cdot)}(\Omega)$ is a Banach space with the norm

$$
\|u\|_{L^{p(\cdot)}(\Omega)}:=\inf \left\{t>0: \rho_{p(\cdot)}(u / t) \leq 1\right\} .
$$

One can compare the functionals \|\|$_{L^{p(\cdot)}(\Omega)}$ and $\rho_{p(\cdot)}()$ using the inequalities

$$
\min \left\{\rho_{p(\cdot)}(u)^{1 / p^{-}}, \rho_{p(\cdot)}(u)^{1 / p^{+}}\right\} \leq\|u\|_{L^{p(\cdot)}(\Omega)} \leq \max \left\{\rho_{p(\cdot)}(u)^{1 / p^{-}}, \rho_{p(\cdot)}(u)^{1 / p^{+}}\right\},
$$

and the unit ball property follows: $\|u\|_{L^{p(\cdot)}(\Omega)} \geq 1$ if and only if $\rho_{p(\cdot)}(u) \geq 1$. Another basic result is Hölder's inequality: if $r(\cdot), p(\cdot)$ and $q(\cdot)$ are exponents satisfying

$$
1 / r(x)=1 / p(x)+1 / q(x),
$$

$\mathcal{L}^{n}$-almost everywhere on $\Omega$, then

$$
\|u v\|_{L^{r(\cdot)}(\Omega)} \leq 2\|u\|_{L^{p(\cdot)}(\Omega)}\|v\|_{L^{q(\cdot)}(\Omega)} \cdot
$$

The Sobolev space $L_{1}^{p(\cdot)}(\Omega)$ is the vector space of those functions $u$ in $L^{p(\cdot)}(\Omega)$ for which their distributional gradient (that we denote by $\nabla u$ if no confusion arises) is also in $L^{p(\cdot)}(\Omega) . L_{1}^{p(\cdot)}(\Omega)$ is also a Banach space with the norm

$$
\|u\|_{L_{1}^{p(\cdot)}(\Omega)}:=\|u\|_{L^{p(\cdot)}(\Omega)}+\|\nabla u\|_{L^{p(\cdot)}(\Omega)} .
$$

Despite the apparent similarity between these spaces and the classical Lebesgue and Sobolev spaces, some properties of the latter are not valid in the variable exponent case: for instance, if $p^{+}$is finite, then for every $\Omega$, the smooth functions are dense in $L^{p(\cdot)}(\Omega)$, see [15]; however, sufficient conditions on $p(\cdot)$ to ensure the density of the smooth functions in $L_{1}^{p(\cdot)}(\Omega)$ even when $\Omega$ is a bounded and smooth domain in $\mathbb{R}^{n}$ are more delicate. The first counterexample of this type was found by Zhikov for variational problems in the 2-dimensional disk: the infimum over the smooth functions of a functional of $p(\cdot)$-Dirichlet type is strictly larger than the infimum of that functional over all the functions in $L_{1}^{p(\cdot)}(\Omega)$, see [21,22]; in those examples, the exponent $p(\cdot)$ is not continuous.

If the domain $\Omega$ has a locally Lipschitz boundary, then a sufficient condition for the aforementioned density was found by Samko and Diening on $\Omega$ provided that $p(\cdot)$ satisfies the log-Hölder condition (see $[4,5,13,14]): p(\cdot)$ is of log-Hölder type if there exists a constant $C_{\log }$ such that

$$
|p(x)-p(y)| \leq C_{\log } / \log (e+1 /|x-y|),
$$

where $|x-y|$ is the Euclidean distance between the elements $x$ and $y$ in $\Omega$. 
To relate log-Hölder continuity with other types of uniform continuity, different moduli of continuity are used: given a continuous function $\phi: \mathbb{R}_{+} \rightarrow \mathbb{R}_{+}$with $\lim _{t \rightarrow 0^{+}} \phi(t)=0$, the exponent $p(\cdot): \Omega \rightarrow[1, \infty[$ is $\phi$-continuous if there exists a constant $C_{\phi}$ such that

$$
|p(x)-p(y)| \leq C_{\phi} \phi(|x-y|)
$$

for every pair of points $\{x, y\}$ in $\Omega$. If $\phi_{1}(t) \leq \phi_{2}(t)$ for $t$ near 0 , then $p(\cdot)$ is $\phi_{2^{-}}$ continuous whenever $p(\cdot)$ is $\phi_{1}$-continuous.

In the quest for the necessity of the log-Hölder condition for the density of the smooth functions on $L_{1}^{p(\cdot)}(\Omega)$, Hästö constructs on the unit disk in $\mathbb{R}^{2}$, a uniformly continuous exponent whose modulus of continuity is slightly weaker than the one associated to the log-Hölder condition, but for which the smooth functions are not dense in $L_{1}^{p(\cdot)}(\Omega)$, see [13]. The modulus used by Hästö is equivalent to

$$
\phi(t)=\log \log (e+1 / t) / \log (e+1 / t)
$$

giving a satisfactory but still partial answer to the problem, see $[6,13,14]$.

In the counterexamples of Zhikov and Hästö, the domain $\Omega$ is the standard smooth domain, namely the unit disk. Beside smoothness, some properties or conditions for the regularity of domains in $\mathbb{R}^{n}$ are as follows:

1. The strong local Lipschitz condition,

2. The segment condition,

3. The uniform cone condition,

4. The cone condition,

5. The weak cone condition, and

6. The measure density condition.

The definition of the first five notions can be found in $[1,20]$. Concerning the sixth condition, a subset $\Omega$ of $\mathbb{R}^{n}$ is said to satisfy the $s$-measure density condition, for some $s>0$, if there exists a positive constant $c$ such that for every $x$ in $\bar{\Omega}$ and each $R$ in ] $0,1]$ one has

$$
c R^{s} \leq\left|B_{R}(x) \cap \Omega\right|
$$

If $s=n$, then we shall simply say that $\Omega$ satisfies the measure density condition.

If $A$ and $B$ are any of the mentioned properties for domains, write $A \Longrightarrow B$ if whenever $\Omega$ is a domain with property $A$, then $\Omega$ also has property $B$. The relations between the mentioned properties can be read from the next picture: 
Strong local Lipschitz condition

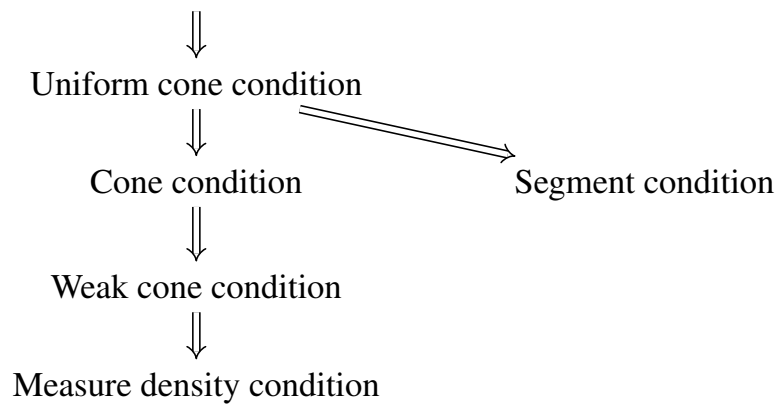

The relation between the first five properties is given in [1]. To illustrate the relations with the measure density condition, we give some examples:

Example 2.1 Consider

$$
\left.\Omega_{1}=\right]-1,1\left[{ }^{2} \sim\{(0,0)\}\right.
$$

the unit square minus the origin in $\mathbb{R}^{2}$. Then $\Omega_{1}$ satisfies the cone condition, but not the segment condition.

Example 2.2 If $K$ is a compact subset of the square ] - 10, $10\left[^{2}\right.$ whose Hausdorff dimension is smaller than 2 , then

$$
\left.\Omega_{2}(K)=\right]-10,10\left[{ }^{2} \sim K\right.
$$

satisfies the measure density condition. In particular, if $K$ is the von Koch Curve (snowflake) whose Hausdorff dimension is $\log 4 / \log 3$, then $\Omega_{2}(K)$ satisfies the measure density condition, but not the weak cone condition, nor the segment condition.

Example 2.3 Let

$$
\Omega_{3}=B_{1}(0) \cap\left\{\left(x_{1}, x_{2}\right): x_{1}>0,\left|x_{2}\right|<\left|x_{1}\right|^{3}\right\} \subset \mathbb{R}^{2} .
$$

Then $\Omega_{3}$ satisfies the segment condition but not the weak cone condition nor the measure density condition.

Another situation where there are similarities and differences between standard and variable exponent Lebesgue and Sobolev spaces is the inclusion problem: Find necessary and sufficient conditions on $p(\cdot), q(\cdot)$ and $\Omega$ such that there exists a constant $C$ so that

$$
\|u\|_{L^{q(\cdot)}(\Omega)} \leq C\|u\|_{L_{1}^{p(\cdot)}(\Omega)},
$$

whenever $u$ is in $L_{1}^{p(\cdot)}(\Omega)$; if that is the case one writes $L_{1}^{p(\cdot)}(\Omega) \hookrightarrow L^{q(\cdot)}(\Omega)$. In this context, Diening obtained the following result: 
Theorem 2.1 (Theorem 5.2 and Corollary 5.3 in [4]) Let $\Omega$ be an open and bounded subset of $\mathbb{R}^{n}$, and suppose that:

1. The boundary of $\Omega$ is locally Lipschitz. ${ }^{1}$

2. The exponent $p(\cdot)$ is log-Hölder continuous, with $1<p^{-} \leq p^{+}<n$.

Conclusion: $L_{1}^{p(\cdot)}(\Omega) \hookrightarrow L^{q(\cdot)}(\Omega)$, where $q(\cdot):=\frac{p(\cdot) n}{n-p(\cdot)}$.

To prove Theorem 2.1, Diening proceeds, roughly speaking, in two steps. In the first step, he obtains the embedding $L_{1}^{p(\cdot)}\left(\mathbb{R}^{n}\right) \hookrightarrow L^{q(\cdot)}\left(\mathbb{R}^{n}\right)$ extending some tools of Potential theory [19] to the case when the exponent $p(\cdot)$ is of log-Hölder type. In the second step, he extends the technique of Extension operators [1] to this setup: as in the case when $p$ is constant, a sufficient condition on $\Omega$ for these purposes is the strong local Lipschitz condition.

The inclusion problem between Sobolev and Hölder spaces has also been extended to variable exponent spaces. As in the classical case, define $C^{0, \alpha(\cdot)}(\Omega)$, the Hölder space of variable exponent $\alpha(\cdot)$ over $\Omega$, where now $\alpha: \Omega \rightarrow] 0,1]$ is a measurable function: given a bounded and continuous function $u$ on $\Omega$ consider its seminorm

$$
[u]_{\alpha(\cdot)}:=\sup _{x \neq y \in \Omega} \frac{|u(x)-u(y)|}{|x-y|^{\alpha(x)}},
$$

so that $C^{0, \alpha(\cdot)}(\Omega)$ is the vector space made up of those $u$ that are bounded, continuous, and for which the seminorm $[u]_{\alpha(\cdot)}$ is finite. $C^{0, \alpha(\cdot)}(\Omega)$ is a Banach space for the norm

$$
\|u\|_{C^{0, \alpha(\cdot)}(\Omega)}:=\|u\|_{\infty}+[u]_{\alpha(\cdot)} .
$$

Using similar ideas and techniques as those used by Diening to obtain Theorem 2.1, Almeida and Samko obtained an inclusion between variable exponent Sobolev and Hölder spaces:

Theorem 2.2 (Theorem 4.7 in [2]) Let $\Omega$ be an open and bounded subset of $\mathbb{R}^{n}$, and suppose that:

1. The boundary of $\Omega$ is locally Lipschitz.

2. The exponent $p(\cdot)$ is log-Hölder continuous, with $n<p^{-}$.

Conclusion: $L_{1}^{p(\cdot)}(\Omega) \hookrightarrow C^{0, \alpha(\cdot)}(\Omega)$, where $\alpha(\cdot):=1-n / p(\cdot)$.

Remark 2.1 By restricting Theorems 2.1 and 2.2 to the case when the exponents are constant, one recovers some of the results given in [1]. However, for constant exponents, a weaker condition on $\Omega$ for the embedding $L_{1}^{p}(\Omega) \hookrightarrow L^{q}(\Omega)$ suffices: the cone condition.

Theorems 2.1 and 2.2 give sufficient conditions both on $\Omega$ and on $\{p(\cdot), \alpha(\cdot)\}$ to achieve the Sobolev and Hölder embeddings with variable exponents, respectively.

\footnotetext{
${ }^{1}$ If $\Omega$ is bounded, then the strong local Lipschitz condition reduces to the locally Lipschitz character of the boundary of $\Omega$.
} 
On the other hand, concerning necessary conditions on the domain $\Omega$ to achieve classical Sobolev and Hölder embeddings, an important progress was achieved by Hajłasz, Koskela and Tuominen, see [11]. Part of their results can be summarized as follows:

Theorem 2.3 (Theorem 1 in [11]) Let $\Omega$ be an open subset of $\mathbb{R}^{n}$, and suppose that either:

1. $L_{1}^{p}(\Omega) \hookrightarrow L^{q}(\Omega)$, where $p<n$ and $q:=\frac{p n}{n-p}$, or

2. $L_{1}^{p}(\Omega) \hookrightarrow C^{0, \alpha}(\Omega)$, where $n<p$ and $\alpha:=1-n / p$.

Conclusion: $\Omega$ satisfies the measure density condition.

\section{Main Results}

As mentioned in Sect. 1, our results could be seen as a mixture of the theorems stated in Sect. 2, in the sense that they provide sufficient conditions on $\{p(\cdot), \alpha(\cdot)\}$ and/or necessary conditions on the domain $\Omega$ for the variable exponent Sobolev and Hölder inclusions to hold. We have:

Theorem 3.1 Let $\Omega$ be an open subset of $\mathbb{R}^{n}$, and suppose that for some $s>1$ :

1. The exponent $p(\cdot)$ is log-Hölder continuous, with $p^{+}<s$.

2. The exponent $q(\cdot):=\frac{p(\cdot) s}{s-p(\cdot)}$ is such that $\frac{1}{q^{-}}<\frac{1}{q^{+}}+\frac{1}{s}$.

3. $L_{1}^{p(\cdot)}(\Omega) \hookrightarrow L^{q(\cdot)}(\Omega)$.

Conclusion: $\Omega$ satisfies the s-measure density condition.

The reader can compare Theorem 3.1 (with $s=n$ ) with Theorem 2.1 and the first part of Theorem 2.3. The reader can also see the diagram in Sect. 2 to understand the gap between the sufficient condition in Theorem 2.1 and the necessary condition in Theorem 3.1. In the next result, the domain $\Omega$ is a bounded domain; as a bonus less restrictions on the exponent $q(\cdot)$ than those in Theorem 3.1 are required:

Theorem 3.2 Let $\Omega$ be an open and bounded subset of $\mathbb{R}^{n}$, and suppose that for some $s>1$ :

1. The exponent $p(\cdot)$ is log-Hölder continuous, with $p^{+}<s$.

2. $L_{1}^{p(\cdot)}(\Omega) \hookrightarrow L^{q(\cdot)}(\Omega)$, where $q(\cdot)=\frac{p(\cdot) s}{s-p(\cdot)}$.

Conclusion: The same as in Theorem 3.1.

Finally, our third main result is a mixture of the conditions in Theorem 2.2 and the second part of Theorem 2.3:

Theorem 3.3 Let $\Omega$ be an open and connected subset of $\mathbb{R}^{n}$, and suppose that for some $s>0$ :

1. The exponent $p(\cdot)$ is log-Hölder continuous, with $s<p^{-}$.

2. $L_{1}^{p(\cdot)}(\Omega) \hookrightarrow C^{0, \alpha(\cdot)}(\Omega)$, where $\alpha(\cdot):=1-s / p(\cdot)$.

Conclusion: The same as in Theorem 3.1.

We refer to the diagram in Sect. 2 to see the gap between the necessary and sufficient conditions on the domain. 


\subsection{Proof of Theorems 3.1, 3.2 and 3.3}

Proof of Theorem 3.1 For a fixed $x$ in $\bar{\Omega}$ define $A_{R}:=B_{R}(x) \cap \Omega$. It is enough to consider the case when $\left|A_{R}\right| \leq 1$, otherwise $\left|A_{R}\right| \geq 1 \geq R^{s}$ whenever $R \leq 1$ and there is nothing to prove; moreover, it is enough to consider $R \leq \frac{1}{4}$. For such an $R$, denote by $\tilde{R}<R$ the smallest real number such that

$$
\left|A_{\tilde{R}}\right|=\frac{1}{2}\left|A_{R}\right|
$$

To prove Theorem 3.1, we need the following Lemma:

Lemma 3.1 Use the same assumptions and notation as in Theorem 3.1. Then there exists a constant $c_{1}>0$ such that for all $x$ in $\bar{\Omega}$ and every $R$ in $\left.] 0,1\right]$, one has the estimate

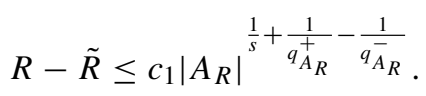

Proof Unless some confusion arises, we omit the domain $\Omega$ in the function spaces and norms. So assume that $L_{1}^{p(\cdot)} \hookrightarrow L^{q(\cdot)}$; then there exists a constant $C_{\text {sob }}>0$ such that whenever $u \in L_{1}^{p(\cdot)}$, one has the inequality

$$
\|u\|_{L^{q(\cdot)}} \leq C_{\mathrm{sob}}\left(\|u\|_{L^{p(\cdot)}}+\|\nabla u\|_{L^{p(\cdot)}}\right) .
$$

For a fixed $x$ in $\bar{\Omega}$ let $u(y):=\phi(y-x)$ be a function of $y \in \Omega$, where $\phi$ is a cut-off function satisfying:

1. $\phi: \mathbb{R}^{n} \rightarrow[0,1]$,

2. $\operatorname{spt} \phi \subset B_{R}(0)$,

3. $\left.\phi\right|_{B_{\tilde{R}}(0)}=1$, and

4. $|\nabla \phi| \leq \tilde{c} /(R-\tilde{R})$ for some constant $\tilde{c}$.

Recall that $x$ is fixed, to deduce the inequalities

$$
\left\|1_{B_{\tilde{R}}}\right\|_{L^{q(\cdot)}} \leq\|u\|_{L^{q(\cdot)}}
$$

and

$$
\begin{aligned}
& \|u\|_{L^{p(\cdot)}}+\|\nabla u\|_{L^{p(\cdot)}} \leq\left\|1_{B_{R}}\right\|_{L^{p(\cdot)}} \\
& +\frac{\tilde{c}}{R-\tilde{R}}\left\|1_{B_{R} \sim B_{\tilde{R}}}\right\|_{L^{p(\cdot)}} \leq \frac{2 \max \{1, \tilde{c}\}}{R-\tilde{R}}\left\|1_{B_{R}}\right\|_{L^{p(\cdot)}} .
\end{aligned}
$$

Combine those inequalities with the Sobolev inequality (3), and

$$
R-\tilde{R} \leq 2 C_{\text {sob }} \max \{1, \tilde{c}\} \frac{\left\|1_{B_{R}}\right\|_{L^{p(\cdot)}}}{\left\|1_{B_{\tilde{R}}}\right\|_{L^{q(\cdot)}}}
$$


follows. Moreover, use Hölder's inequality (1)

$$
\left\|1_{B_{R}}\right\|_{L^{p(\cdot)}} \leq 2\left\|1_{B_{R}}\right\|_{L^{s}}\left\|1_{B_{R}}\right\|_{L^{q(\cdot)}}
$$

and the basic estimates between norm and semimodular in (4), to infer that

$$
\begin{aligned}
R-\tilde{R} & \leq 4 C_{\text {sob }} \max \{1, \tilde{c}\}\left|A_{R}\right|^{\frac{1}{s}} \frac{\left\|1_{B_{R}}\right\|_{L^{q(\cdot)}}}{\left\|1_{B_{\tilde{R}}}\right\|_{L^{q(\cdot)}}} \\
& \leq 4 C_{\text {sob }} \max \{1, \tilde{c}\}\left|A_{R}\right|^{\frac{1}{s}} \frac{\left|A_{R}\right|^{\frac{1}{q_{A_{R}}^{+}}}}{\mid \frac{1}{q_{\tilde{A}}^{-}}} \\
& =4 C_{\text {sob }} \max \{1, \tilde{c}\} 2^{\frac{1}{q_{A}^{-}}}\left|A_{R}\right|^{\frac{1}{s}+\frac{1}{q_{A_{R}}^{+}}-\frac{1}{q_{A}^{-}}} \\
& \leq 4 C_{\text {sob }} \max \{1, \tilde{c}\} 2^{\frac{1}{q^{-}}}\left|A_{R}\right|^{\frac{1}{s}+\frac{1}{q_{A_{R}}^{+}}-\frac{1}{q_{A_{R}}^{-}}},
\end{aligned}
$$

as claimed.

To conclude the proof of Theorem 3.1, given $x$ in $\bar{\Omega}$ and $R$ in ]0, $\left.\frac{1}{4}\right]$, construct the sequence $\left\{R_{i}\right\}$ by setting $R_{0}:=R$, and then define $R_{i+1}:=\tilde{R}_{i}$ inductively for $i \geq 0$. It follows that

$$
\left|A_{R_{i}}\right|=\frac{1}{2^{i}}\left|A_{R}\right|
$$

with $\lim _{i \rightarrow \infty} R_{i}=0$.

With those ingredients in Lemma 3.1, one observes that

$$
R_{i}-R_{i+1} \leq c_{1}\left|A_{R_{i}}\right|^{\frac{1}{s}+\frac{1}{q_{A_{R_{i}}}^{+}}-\frac{1}{q_{A_{R_{i}}}^{-}}} \leq c_{1}\left|A_{R_{i}}\right|^{\frac{1}{s}+\frac{1}{q_{A_{R}}^{+}}-\frac{1}{q_{A_{R}}^{-}}}=c_{1} \frac{\left|A_{R}\right|^{\eta_{R}}}{2^{i \eta_{R}}}
$$

where the abbreviation $\eta_{R}:=\frac{1}{s}+\frac{1}{q_{A_{R}}^{+}}-\frac{1}{q_{A_{R}}^{-}}$has been used.

Note that $\eta_{R} \geq \eta:=\frac{1}{s}+\frac{1}{q^{+}}-\frac{1}{q^{-}}>0$, to deduce, thanks to the previous observations, that

$$
\begin{aligned}
R & =\sum_{i=0}^{\infty}\left(R_{i}-R_{i+1}\right) \leq c_{1}\left|A_{R}\right|^{\eta_{R}} \sum_{i=0}^{\infty} 2^{-i \eta_{R}} \\
& =c_{1}\left|A_{R}\right|^{\eta_{R}} \frac{1}{1-2^{-\eta_{R}}} \leq \max \left\{1, \frac{c_{1}}{1-2^{-\eta}}\right\}\left|A_{R}\right|^{\eta_{R}} .
\end{aligned}
$$


Moreover, since $c_{2}:=1 / \max \left\{1, \frac{c_{1}}{1-2^{-\eta}}\right\} \leq 1$ one has

$$
\left|A_{R}\right| \geq c_{2}^{1 / \eta_{R}} R^{1 / \eta_{R}} \geq c_{2}^{1 / \eta} R^{1 / \eta_{R}}=c_{2}^{1 / \eta} R^{s} R^{\beta_{R} / \eta_{R}},
$$

where $\beta_{R}:=1-s \eta_{R}$.

From (6), one sees that if a positive lower bound for $R^{\beta_{R} / \eta_{R}}$ is provided, the proof of Theorem 3.1 is finished. To achieve such a lower bound, the log-Hölder continuity of $p(\cdot)$ will be used: by the hypotheses on $p(\cdot)$ and $q(\cdot)$ one has that $q(\cdot)$ is log-Hölder continuous as well, hence

$$
|q(z)-q(y)| \leq \frac{C_{\log }}{\log (e+1 /|z-y|)}
$$

taking the supremum over pairs of points in $A_{R}$ one gets

$$
q_{A_{R}}^{+}-q_{A_{R}}^{-} \leq \frac{C_{\log }}{\log (1 /(2 R))}
$$

or

$$
\log \left(1 /(2 R)^{q_{A_{R}}^{+}-q_{A_{R}}^{-}}\right) \leq C_{\log }
$$

therefore

$$
R^{q_{A_{R}}^{+}-q_{A_{R}}^{-}} \geq \frac{e^{-C_{\log }}}{2^{q_{A_{R}}^{+}-q_{A_{R}}^{-}}} \geq \frac{e^{-C_{\log }}}{2^{\left(q^{+}-q^{-}\right)}} .
$$

But

$$
R^{\frac{\beta_{R}}{\eta_{R}}} \geq R^{\frac{\beta_{R}}{\eta}}=R^{\frac{s\left(q_{A_{R}}^{+}-q_{A_{R}}^{-}\right)}{\eta q_{A_{R}}^{+} q_{A_{R}}^{-}}} \geq\left(R^{\left.q_{A_{R}}^{+}-q_{A_{R}}^{-}\right)^{s / \eta\left(q^{-}\right)^{2}}},\right.
$$

hence using (7) the required bound

$$
R^{\frac{\beta_{R}}{\eta_{R}}} \geq\left(\frac{e^{-C_{\log }}}{2^{\left(q^{+}-q^{-}\right)}}\right)^{s / \eta\left(q^{-}\right)^{2}}=: c_{3}>0
$$

follows, and the uniform estimate $c R^{s} \leq\left|B_{R}(x) \cap \Omega\right|$ is obtained, where $c:=c_{2}^{1 / \eta} c_{3}$.

Proof of Theorem 3.2 We use a notation similar to that in Theorem 3.1. Since $q(\cdot)$ is log-Hölder continuous, $q(\cdot)$ is uniformly continuous, and one can extend $q(\cdot)$ to $\bar{\Omega}$. Since $\bar{\Omega}$ is compact, there is a finite cover $\left\{O_{\alpha}: \alpha\right.$ in $\left.\Lambda\right\}$ of $\bar{\Omega}$ by open sets such that

$$
\eta_{\alpha}:=\frac{1}{s}+\frac{1}{q_{O_{\alpha}}^{+}}-\frac{1}{q_{O_{\alpha}}^{-}}>0 .
$$


Let $\lambda$ be some Lebesgue number of the covering $\left\{O_{\alpha}\right\}$, and consider $R$ such that $R \leq \min \{1 / 4, \lambda / 2\}$. Fix some $x$ in $\Omega$, note that $B_{R}(x) \subset O_{\alpha}$ for some $\alpha \in \Lambda$, to define the sequence $\left\{R_{i}\right\}$ in the same way as in the proof of Theorem 3.1: hence by (5)

$$
\begin{gathered}
R \leq c_{1}\left|A_{R}\right|^{\eta_{R}} \frac{1}{1-2^{-\eta_{R}}} \\
\leq \max \left\{1, \frac{c_{1}}{1-2^{-\eta_{\alpha}}}\right\}\left|A_{R}\right|^{\eta_{R}},
\end{gathered}
$$

where $\eta_{R} \geq \eta_{\alpha}>0$. Proceeding in a similar way as in Theorem 3.1, one obtains

$$
\left|A_{R}\right| \geq c_{2, \alpha}^{1 / \eta_{\alpha}} R^{s} R^{\beta_{R} / \eta_{R}}
$$

where $c_{2, \alpha}:=1 / \max \left\{1, \frac{c_{1}}{1-2^{-\eta_{\alpha}}}\right\}$; also for each $\alpha$ in $\Lambda$ one has

$$
R^{\beta_{R} / \eta_{R}} \geq R^{\beta_{R} / \eta_{\alpha}} \geq\left(\frac{e^{-C_{\log }}}{2^{\left(q^{+}-q^{-}\right)}}\right)^{s / \eta_{\alpha}\left(q^{-}\right)^{2}}=: c_{3, \alpha}
$$

Putting things together the claimed estimate $\left|A_{R}\right| \geq c R^{s}$ follows, where

$$
c:=\min \left\{c_{2, \alpha}^{1 / \eta_{\alpha}} c_{3, \alpha}: \alpha \text { in } \Lambda\right\}
$$

From Theorem 3.2, we deduce:

Corollary 3.1 Let $\Omega$ be an open and bounded subset of $\mathbb{R}^{n}$, and suppose that:

1. The exponent $p(\cdot)$ is log-Hölder continuous.

2. $L_{1}^{p(\cdot)}(\Omega) \hookrightarrow L^{q(\cdot)}(\Omega)$, where $q(\cdot)$ is measurable and such that $q(x)>p(x)$ whenever $x$ is in $\Omega$.

Conclusion: There exists a constant $c>0$ such that for all $x$ in $\Omega$ and all $R$ in ]0, 1], one has the estimate

$$
c R^{t} \leq\left|B_{R}(x) \cap \Omega\right|
$$

whenever

$$
t:=\sup \left\{\frac{p(x) q(x)}{q(x)-p(x)}: x \text { in } \Omega\right\}
$$

is finite. 
Proof Introduce the function

$$
Q(\cdot):=\frac{p(\cdot) t}{t-p(\cdot)}
$$

and note that $1 \leq Q(\cdot) \leq q(\cdot)$, hence

$$
L_{1}^{p(\cdot)} \hookrightarrow L^{q(\cdot)} \hookrightarrow L^{Q(\cdot)}
$$

Moreover, since $p(\cdot)<q(\cdot)$ one has that

$$
t \geq \frac{p^{-} q^{+}}{q^{+}-p^{-}}>1
$$

with $p^{+}<t$. One can use $Q(\cdot)$ instead of $q(\cdot)$ and $t$ instead of $s$ in the hypotheses of Theorem 3.2: the result follows.

Proof of Theorem 3.3 We use a notation similar to that in Theorems 3.1 and 3.2. Fix $x$ in $\bar{\Omega}$ and some $R \leq \frac{1}{4}$; we assume that $\Omega \sim B_{R}(x) \neq \emptyset$, since otherwise $\left|A_{R}\right|=|\Omega|$. Let $u(y):=\phi(y-x)$ be a function of $y \in \Omega$, where $\phi$ is a cut-off function satisfying:

1. $\phi: \mathbb{R}^{n} \rightarrow[0,1]$,

2. $\operatorname{spt} \phi \subset B_{R}(0)$,

3. $\phi(0)=1$, and

4. $|\nabla \phi| \leq \tilde{c} / R$ for some constant $\tilde{c}$.

The hypothesis $L_{1}^{p(\cdot)} \hookrightarrow C^{0, \alpha(\cdot)}$ entails that whenever $u \in L_{1}^{p(\cdot)}$ one has that

$$
|u(y)-u(z)| \leq C_{\mathrm{sob}}\|u\|_{L_{1}^{p(\cdot)}}|y-z|^{1-s / p(y)},
$$

for every pair of points $\{y, z\}$ in $\Omega$. In (8) choose $y=x$ and $z \in\left(\Omega \sim B_{R}(x)\right) \cap$ $B_{2 R}(x):{ }^{2}$ one gets

$$
1 \leq C_{\text {sob }}\|u\|_{L_{1}^{p(\cdot)}}|x-z|^{1-s / p(x)} .
$$

On the other hand

$$
\|u\|_{L_{1}^{p(\cdot)}}=\|u\|_{L^{p(\cdot)}}+\|\nabla u\|_{L^{p(\cdot)}} \leq \frac{1+\tilde{c}}{R}\left\|1_{B_{R}(x)}\right\|_{L^{p(\cdot)}} \leq \frac{1+\tilde{c}}{R}\left|A_{R}\right|^{1 / p_{A_{R}}^{+}}
$$

and

$$
|x-z|^{1-s / p(x)} \leq(2 R)^{1-s / p(x)} \leq(2 R)^{1-s / p_{A_{R}}^{-}}
$$

\footnotetext{
$\overline{2 \text { Since } \Omega \text { is connected, we have }}\left(\Omega \sim B_{R}(x)\right) \cap B_{2 R}(x) \neq \emptyset$.
} 
Using (10) and (11) in (9)

$$
1 \leq C_{\mathrm{sob}} \frac{1+\tilde{c}}{R}\left|A_{R}\right|^{1 / p_{A_{R}}^{+}}(2 R)^{1-s / p_{A_{R}}^{-}} \leq 2 C_{\mathrm{sob}}(1+\tilde{c})\left|A_{R}\right|^{1 / p_{A_{R}}^{+}} R^{-s / p_{A_{R}}^{-}}
$$

follows, therefore

$$
\left|A_{R}\right| \geq\left(\frac{1}{2 C_{\mathrm{sob}}(1+\tilde{c})}\right)^{p_{A_{R}}^{+}} R^{s \frac{p_{A_{R}}^{+}}{p_{A_{R}}^{-}}} \geq C^{p^{+}} R^{s} R^{s \frac{p_{A_{R}}^{+}-p_{A_{R}}^{-}}{p_{A_{R}}^{-}}},
$$

where $C=\min \left\{1,1 /\left(2 C_{\text {sob }}(1+\tilde{c})\right)\right\}$. Finally, a positive lower bound for $R^{s\left(p_{A_{R}}^{+}-p_{A_{R}}^{-}\right) / p_{A_{R}}^{-}}$is required; use that $p(\cdot)$ is log-Hölder continuous to obtain the desired estimate (see inequality (7) in the proof of Theorem 3.1):

$$
R^{s \frac{p_{A_{R}}^{+}-p_{A_{R}}^{-}}{p_{A_{R}}^{-}}} \geq\left(R^{\left(p_{A_{R}}^{+}-p_{A_{R}}^{-}\right)}\right)^{s / p^{-}} \geq\left(\frac{e^{-C_{\mathrm{log}}}}{2^{\left(p^{+}-p^{-}\right)}}\right)^{s / p^{-}} .
$$

\section{Consequences and Questions}

In all the results in this work, the measure density condition on the domain $\Omega$ is necessary. With the help of Federer's co-area formula, we will characterize some domains that do not satisfy the measure density condition. Such characterization together with the theorems in this work provides a simpler proof of results from [1]; at the same time, an extension of those results to Sobolev and Hölder spaces with variable exponents follows.

According to [1] the domain $\Omega$ has a cusp of exponential sharpness at its boundary point $x_{0}$ if for every real number $s$ one has

$$
\lim _{R \rightarrow 0_{+}} \frac{\mathcal{H}^{n-1}\left(\Omega \cap \partial B_{R}\left(x_{0}\right)\right)}{R^{s}}=0
$$

Using such notion, the following result is related with Theorem 4.48 in [1]:

Theorem 4.1 Let $\Omega$ be an open subset of $\mathbb{R}^{n}$, and assume that $\Omega$ has a cusp of exponential sharpness at some point of its boundary. Then:

1. $L_{1}^{p(\cdot)}(\Omega)$ is not embedded in $L^{q(\cdot)}$ for those $p(\cdot)$ and $q(\cdot)$ satisfying the conditions in Theorem 3.1, and

2. $L_{1}^{p(\cdot)}(\Omega)$ is not embedded in $C^{0, \alpha(\cdot)}$ for those $p(\cdot)$ and $\alpha(\cdot)$ satisfying the conditions in Theorem 3.3.

Proof For a fixed $x$ in $\bar{\Omega}$ and a fixed $R$ in ]0, 1], consider the map $\pi: \Omega \cap B_{R}(x)=$ $A_{R} \rightarrow[0, R]$ given by $\pi(y)=|y-x|$, and note that if $y \neq x$, then $J_{1} \pi(y)=1$, 
where $J_{1} \pi=|\nabla \pi|$ is the 1-dimensional Jacobian of $\pi$. By Federer's co-area formula (see [7], Paragraph 3.2.11)

$$
\int_{A_{R}} J_{1} \pi(y) \mathrm{d} \mathcal{L}^{n}(y)=\int_{0}^{R}\left\{\int_{\pi^{-1}(r)} \mathrm{d} \mathcal{H}^{n-1}(z)\right\} \mathrm{d} r,
$$

or

$$
\left|A_{R}\right|=\mathcal{L}^{n}\left(A_{R}\right)=\int_{0}^{R} \mathcal{H}^{n-1}\left(\Omega \cap \partial B_{r}(x)\right) \mathrm{d} r
$$

where $\partial B_{r}(x)$ is the boundary of $B_{r}(x)$, and $\mathcal{H}^{k}$ is the $k$-dimensional Hausdorff measure on $\mathbb{R}^{n}$. Hence whenever $R$ and $s$ are positive real numbers, the inequality

$$
\frac{\left|A_{R}\right|}{R^{s}} \leq \int_{0}^{R} \frac{\mathcal{H}^{n-1}\left(\Omega \cap \partial B_{r}(x)\right)}{r^{s}} \mathrm{~d} r
$$

follows from (12).

Assume that $\Omega$ has a cusp of exponential sharpness at $x_{0}$; then for each $s$ there exists some $\tilde{R}$ such that

$$
\frac{\mathcal{H}^{n-1}\left(\Omega \cap \partial B_{r}\left(x_{0}\right)\right)}{r^{s}} \leq 1
$$

whenever $r \leq \tilde{R}$. Moreover, if $R \leq 1$, then

$$
\int_{0}^{R} \frac{\mathcal{H}^{n-1}\left(\Omega \cap \partial B_{r}\left(x_{0}\right)\right)}{r^{s}} \mathrm{~d} r=\int_{0}^{1} \mathbf{1}_{[0, R]}(r) \frac{\mathcal{H}^{n-1}\left(\Omega \cap \partial B_{r}\left(x_{0}\right)\right)}{r^{s}} \mathrm{~d} r
$$

with

$$
\lim _{R \rightarrow 0_{+}} \mathbf{1}_{[0, R]}(r) \frac{\mathcal{H}^{n-1}\left(\Omega \cap \partial B_{r}\left(x_{0}\right)\right)}{r^{s}}=0
$$

from (14), and by Lebesgue's dominated convergence

$$
\lim _{R \rightarrow 0_{+}} \int_{0}^{R} \frac{\mathcal{H}^{n-1}\left(\Omega \cap \partial B_{r}\left(x_{0}\right)\right)}{r^{s}} \mathrm{~d} r=0 .
$$

Therefore using (13) we conclude that $\Omega$ does not satisfy the $s$-measure density condition.

We conclude the article with two open problems. The first one is motivated by Theorem 4.1 and the discussion in Sect. 2: 
Question 4.1 Are embeddings between variable exponent Sobolev and/or Hölder spaces possible if the modulus of continuity of the exponents is weaker than logHölder, for example, given by the modulus in (2)? If so, for which regularity of the domain $\Omega$ might this happen?

The second problem is motivated by Theorem 3.2 and another notion of regularity condition of the exponent: we say that the exponent $p(\cdot)$ is log-Hölder continuous at infinity, if there exist constants $C_{\infty}$ and $p_{\infty}$ such that for all $x \in \Omega$,

$$
\left|p(x)-p_{\infty}\right| \leq C_{\infty} / \log (e+|x|) .
$$

Question 4.2 Can we replace the assumption $\frac{1}{q^{-}}<\frac{1}{q^{+}}+\frac{1}{s}$ in Theorem 3.1 by the assumption that $p(\cdot)(\operatorname{or} q(\cdot))$ is log-Hölder continuous at infinity?

Acknowledgements N. K. thanks DST-SERB (Project PDF/2016/000328) for financial support. P. G. has been supported by NCN grant 2018/02/X/ST1/02133. All the Authors would like to thank the reviewer for his/her valuable comments on the manuscript.

Open Access This article is licensed under a Creative Commons Attribution 4.0 International License, which permits use, sharing, adaptation, distribution and reproduction in any medium or format, as long as you give appropriate credit to the original author(s) and the source, provide a link to the Creative Commons licence, and indicate if changes were made. The images or other third party material in this article are included in the article's Creative Commons licence, unless indicated otherwise in a credit line to the material. If material is not included in the article's Creative Commons licence and your intended use is not permitted by statutory regulation or exceeds the permitted use, you will need to obtain permission directly from the copyright holder. To view a copy of this licence, visit http://creativecommons.org/licenses/by/4.0/.

\section{References}

1. Adams, R.A., Fournier, J.: Sobolev Spaces, 2nd edn. Elsevier, Amsterdam (2005)

2. Almeida, A., Samko, S.: Pointwise inequalities in variable Sobolev spaces and applications. Z. Anal. Anwend. 26, 179-193 (2007)

3. Cruz-Uribe, D., Fiorenza, A.: Variable Lebesgue Spaces (Foundations and Harmonic Analysis), Birkhäuser (2013)

4. Diening, L.: Riesz potential and Sobolev embeddings on generalized Lebesgue and Sobolev spaces $L^{p(\cdot)}$ and $W^{k, p(\cdot)}$. Math. Nach. 268, 31-43 (2004)

5. Diening, L., Harulehto, P., Hästö, P., Rŭẑiĉka, M.: Lebesgue and Sobolev Spaces with Variable Exponents. Lecture Notes in Mathematics. Springe, Heidelberg (2011)

6. Diening, L., Hästö, P., Nekvinda, A.: Open problems in variable exponent Lebesgue and Sobolev spaces. In: Proceeding of the International Conference Function Spaces, Differential Operators and Nonlinear Analysis, Milovy, Czech Republic. Institute of Mathematics of the Academy of Sciences of the Czech Republic, Prague (2004), pp 38-58

7. Federer, H.: Geometric Measure Theory. Springer, New York (1969)

8. Futamura, T., Mizuta, Y., Shimomura, T.: Sobolev embeddings for variable exponent Riesz potentials on metric spaces. Ann. Acad. Sci. Fenn. Math. 31, 495-522 (2006)

9. Gaczkowski, M., Górka, P.: Sobolev spaces with variable exponents on Riemannian manifolds. Nonlinear Anal. 92, 47-59 (2013)

10. Gaczkowski, M., Górka, P., Pons, D.J.: Sobolev spaces with variable exponents on complete manifolds. J. Funct. Anal. 270, 1379-1415 (2016)

11. Hajłasz, P., Koskela, P., Tuominen, H.: Sobolev embeddings, extensions and measure density condition. J. Funct. Anal. 254, 1217-1234 (2008)

12. Harjulehto, P., Hästö, P., Latvala, V.: Sobolev embeddings in metric measure spaces with variable dimension. Math. Z. 254, 591-609 (2006) 
13. Hästö, P.: Counter-Examples of Regularity in Variable Exponente Sobolev Spaces, Contemporary Mathematics, The $p$-Harmonic Equation and Recent Advances in Analysis, Contemporary Mathematics, pp. 133-143. American Mathematical Society, Providence (2005)

14. Hästö, P.: On the density of continuous functions in variable exponent Sobolev space. Rev. Mat. Iberoam. 23(1), 213-234 (2007)

15. Kováčik, O., Rákosnik, J.: On spaces $L^{p(\cdot)}$ and $W^{k, p(\cdot)}$. Czechoslovak Math. J. 41, 592-618 (1991)

16. Li, F., Li, Z., Pi, L.: Variable exponent functionals in image restoration. Appl. Math. Comput. 216(3), 870-882 (2010)

17. Mizuta, Y., Shimomura, T.: Continuity of Sobolev functions of variable exponent on metric spaces. Proc. Jpn. Acad. Ser. A 80(6), 96-99 (2004)

18. Rŭẑiĉka, M.: Electrorheological Fluids: Modeling and Mathematical Theory. Springer-verlag, Berlin (2000)

19. Stein, E.M.: Singular Integrals and Differentiability Properties of Functions. Princeton University Press, Princeton (1970)

20. Wloka, J.: Partial Differential Equations, Cambidge University Press, 1987 (Translation of Partielle Differerentialgleichungen, B.G Teubner, Stuttgart, 1982, by C.B. and M. J. Thomas)

21. Zhikov, V.V.: Averaging of functionals of the calculus of variations and elasticity theory. Math. USSRIzv. 29, 33-66 (1987)

22. Zhikov, V.V.: On Lavrientev’s Phenomenon. Russ. J. Math. Phys. 3, 219-269 (1995)

Publisher's Note Springer Nature remains neutral with regard to jurisdictional claims in published maps and institutional affiliations. 